\title{
The Efficacy of Chest X-Ray for the Diagnosis and Follow-up in Young Adult COVID-19 Patients with Mild Dyspnea No Comorbid Diseases
}

ferhat cuce $^{1}$, deniz dogan ${ }^{1}$, ugur bozlar ${ }^{1}$, mustafa tasar $^{1}$, ervin gjoni ${ }^{1}$, tunahan ayaz ${ }^{1}$, derya $\mathrm{kinl}^{2}$, umit savasc $^{1}$, and Oktay Sarl ${ }^{3}$

${ }^{1}$ Gulhane Training and Research Hospital

${ }^{2}$ Health Science University, Gulhane Training, and Research Hospital, Ankara, Turkey

${ }^{3}$ University of Health Sciences Gülhane Health Sciences Institute

June 8, 2021

\begin{abstract}
Purpose: We investigated whether Chest X-Ray (CXR) could replace CT modality in the diagnosis and during the treatment of young adult COVID-19 patients with mild dyspnea with no comorbid diseases. Materials and Method: This retrospective study involved an examination of the records of a total of 956 patients hospitalized between March 1 and May 15, 2020. The study included a total of 64 patients, aged 21-60 years with mild dyspnea with no comorbid diseases and with COVID-19 infection confirmed by a polymerase chain reaction, who underwent a CXR at admission and CT imaging within 24 hours. The first CXR and CT images at the time of admission were evaluated in terms of lesions and localization. The clinical-radiological course of the diseases with CXR were also statistically evaluated. Results: CT was normal in 18/64 (28.2\%) patients, all of whom also had normal CXR. The rest of the patients $46 / 64(71.8 \%)$ with an abnormal CT, the CXR was normal in $18 / 46$ (39.1\%) and abnormal in $28 / 46(60.9 \%)$. The time between the onset of complaints and admission to the hospital in patients with abnormal and normal CXR was $3.5 \pm 2.3$ days and $2.1 \pm 1.1$, which was statistically significant $(\mathrm{p}=0.004)$. The hospital stay duration of the patient with abnormal and normal CXR was $9.6 \pm 3.5$ and $9.5 \pm 3.4(\mathrm{p}=0.928)$, respectively, and was not statistically significant. Conclusion: CXR could be used in the diagnosis and follow-up of young adult COVID 19 patients with mild dyspnea no comorbid disease. In the case of early admission to the hospital, there is not a significant difference between using CXR or CT in the management of these patients. Therefore, the use of CXR in these patients groups will reduce the burden of $\mathrm{CT}$ units in pandemic conditions with limited resources.
\end{abstract}

\section{Hosted file}

clinical practice 11.edited.docx available at https://authorea.com/users/418709/articles/ 525387-the-efficacy-of-chest-x-ray-for-the-diagnosis-and-follow-up-in-young-adultcovid-19-patients-with-mild-dyspnea-no-comorbid-diseases 


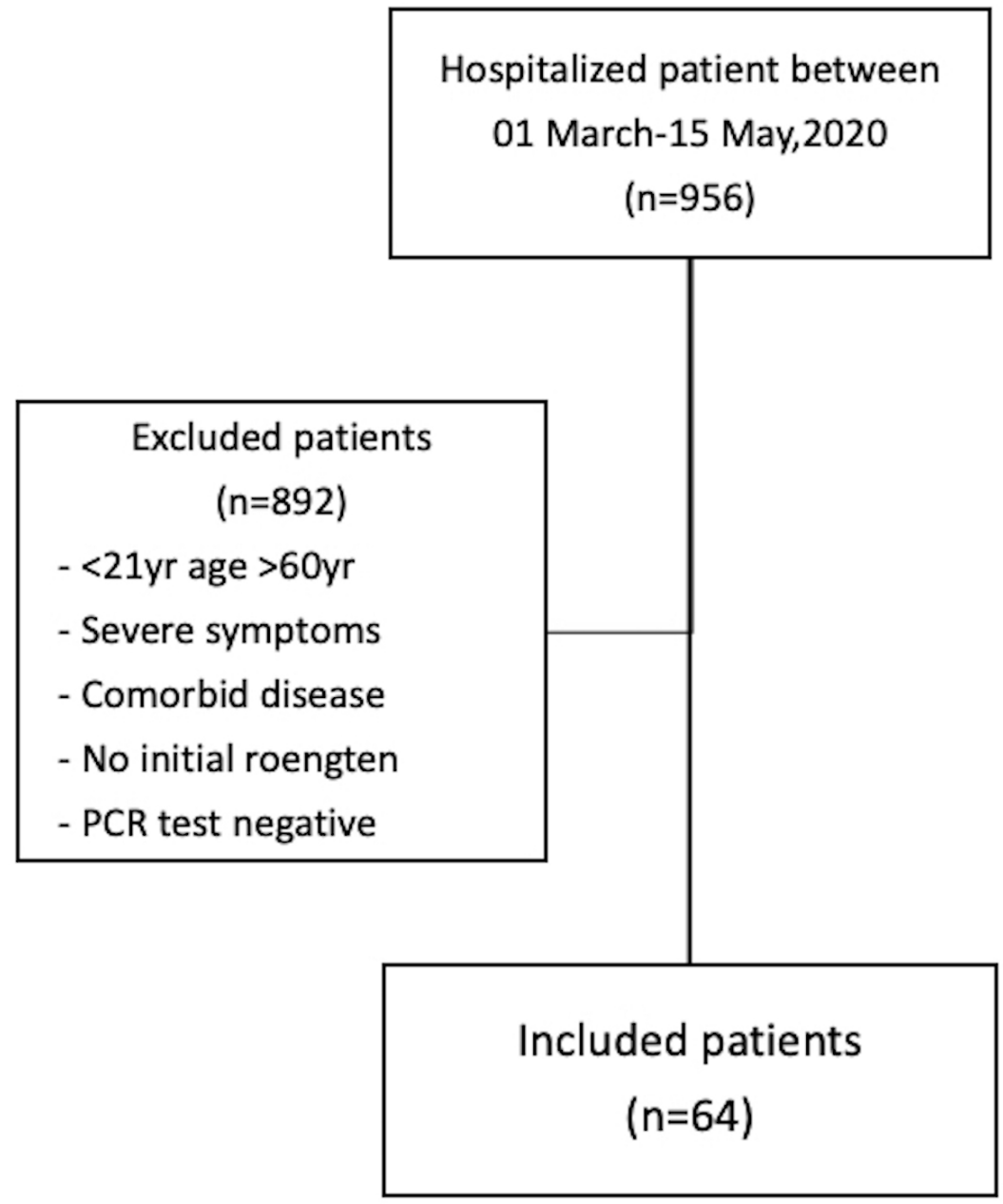



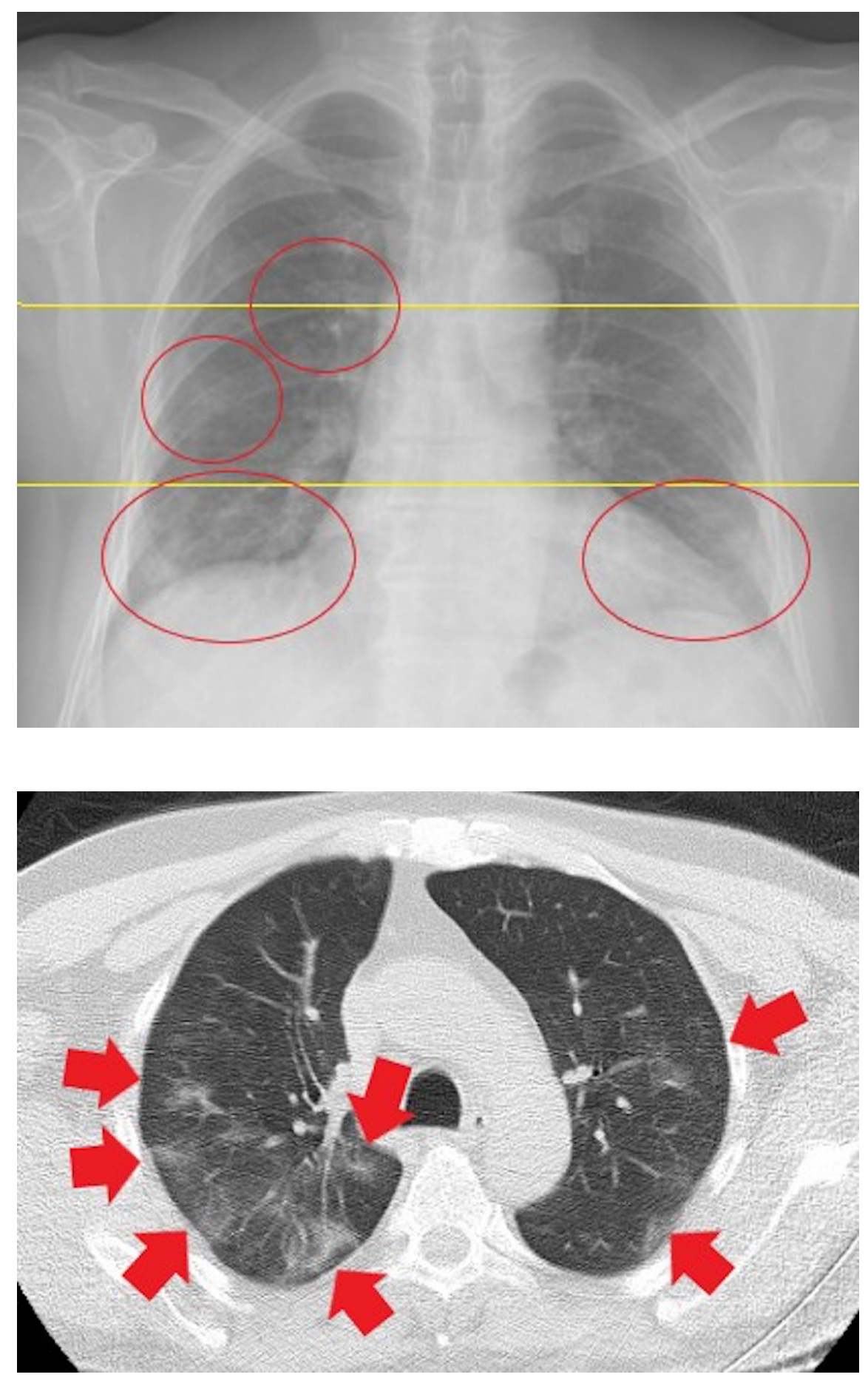

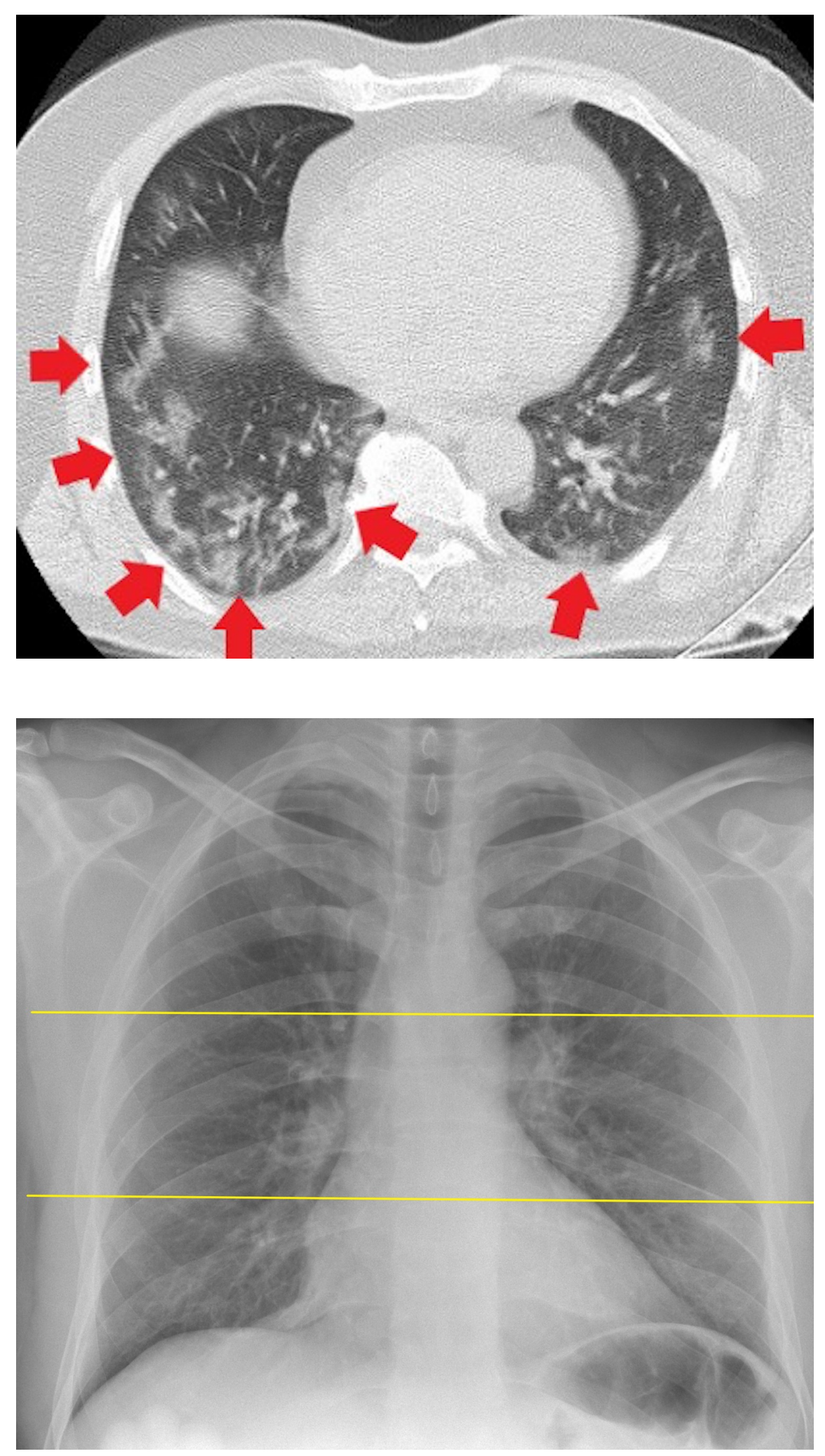

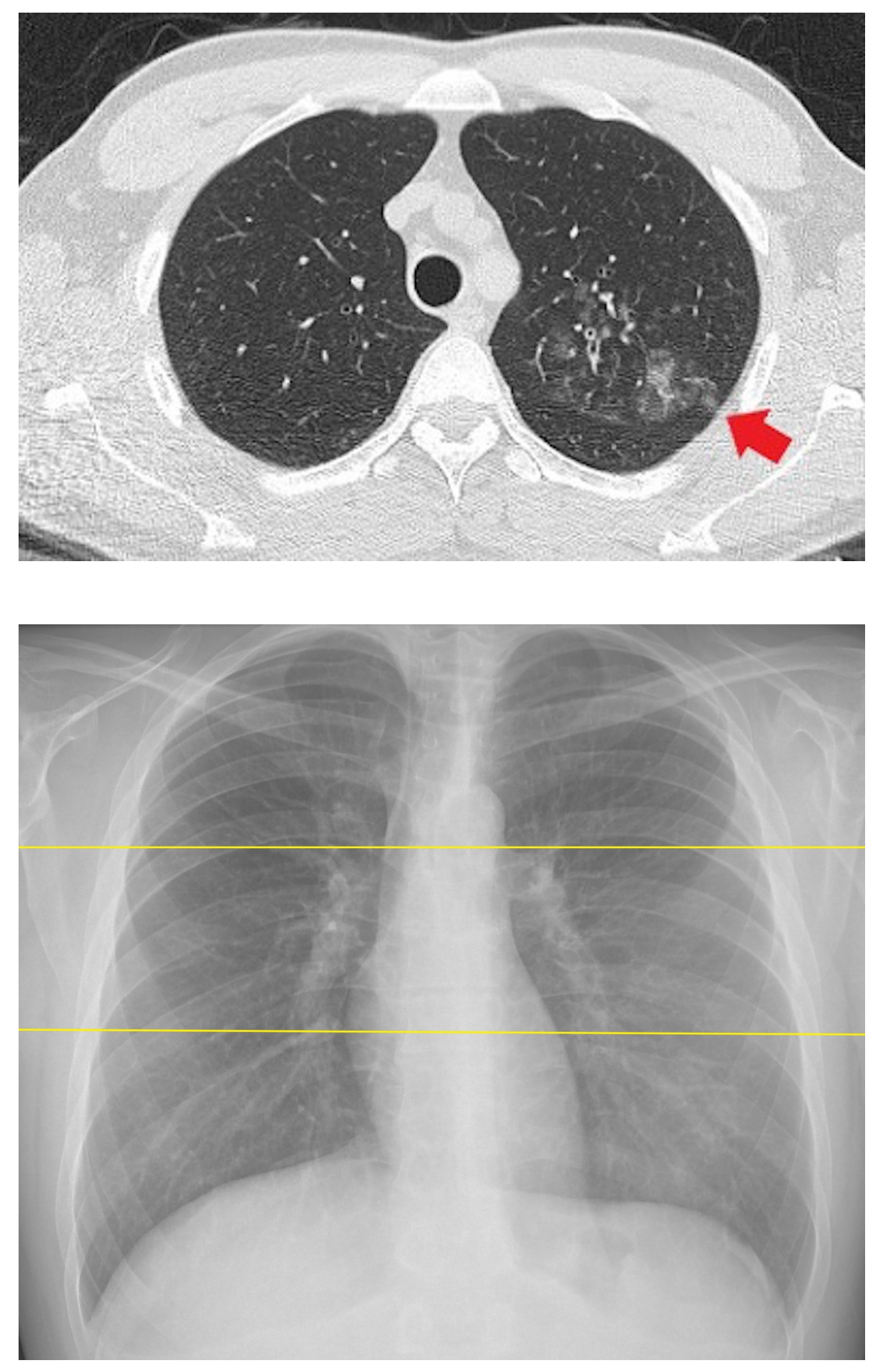


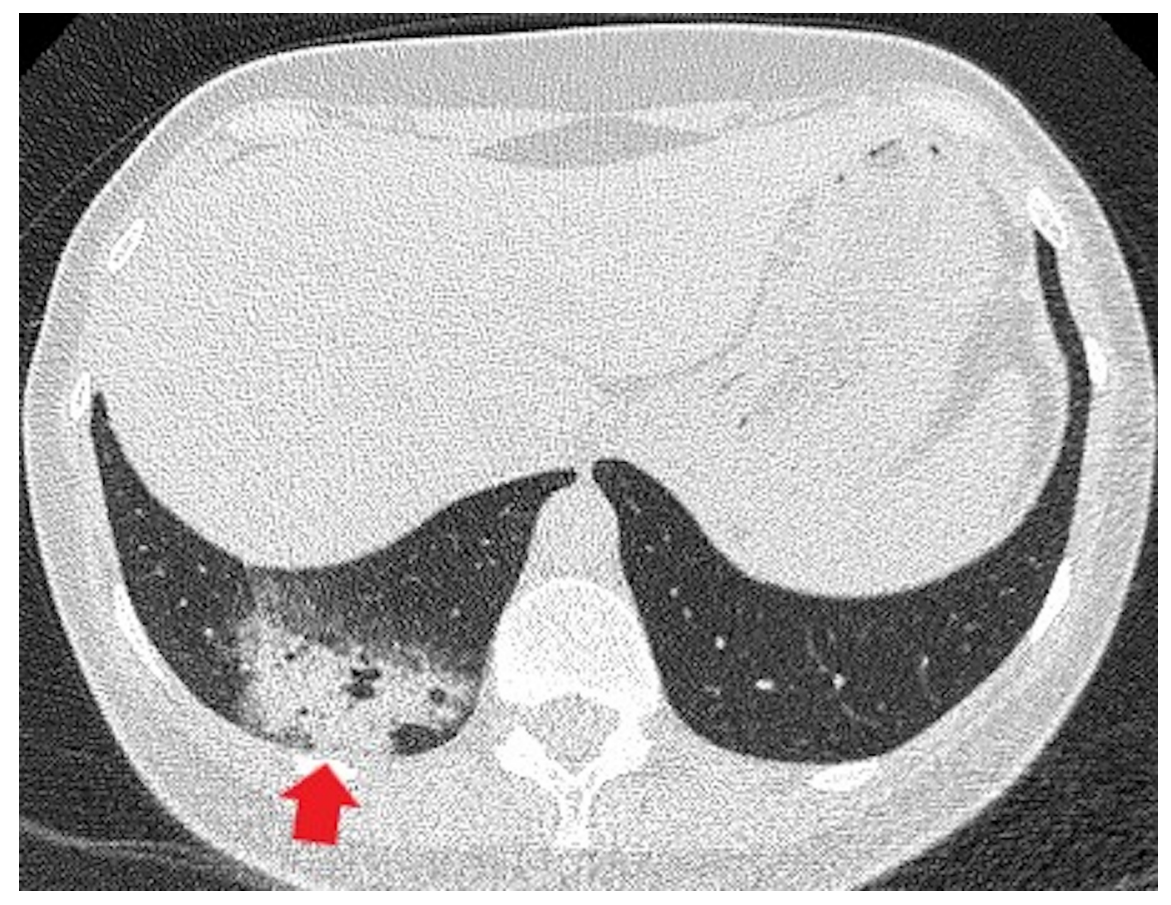




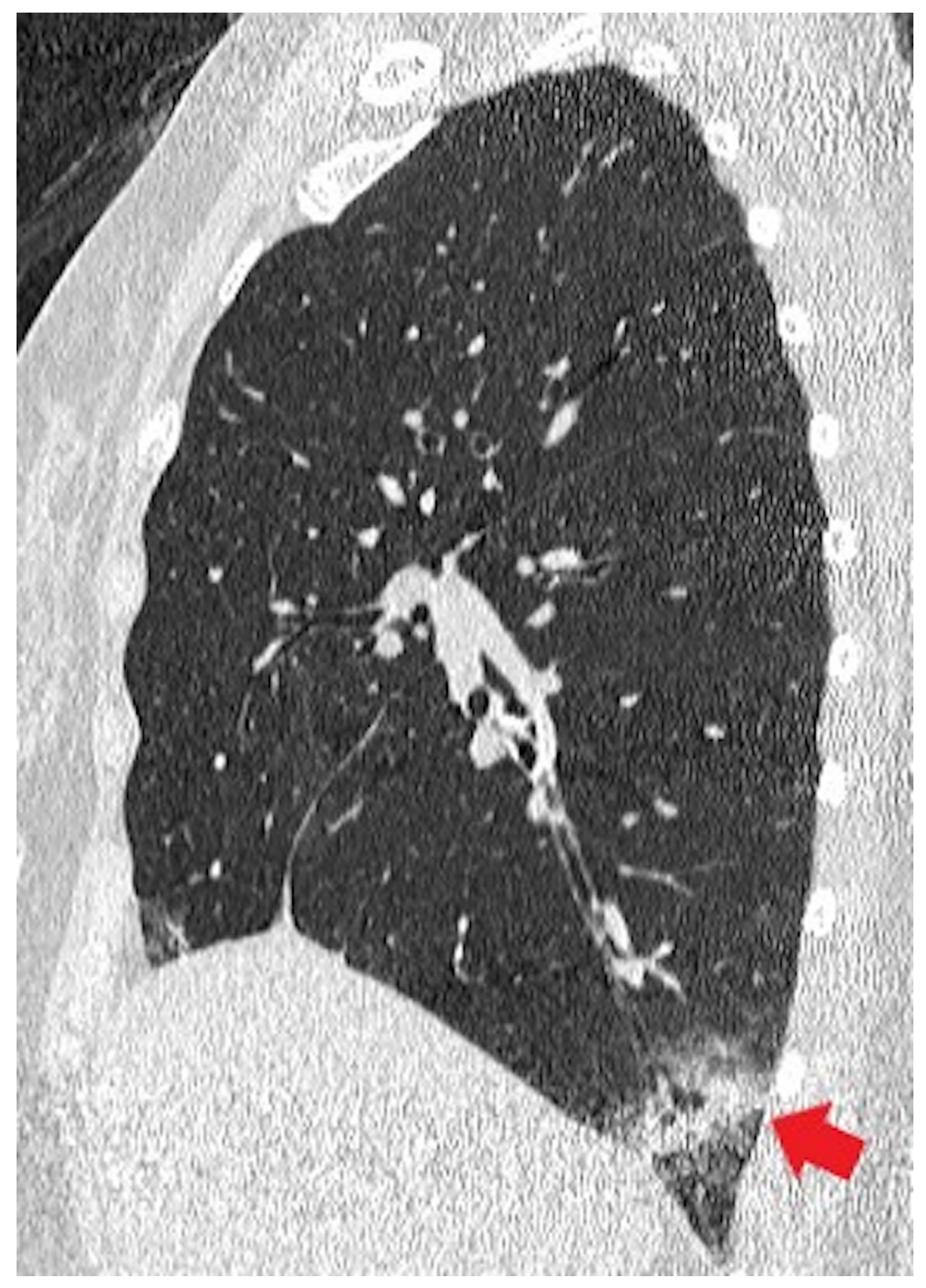



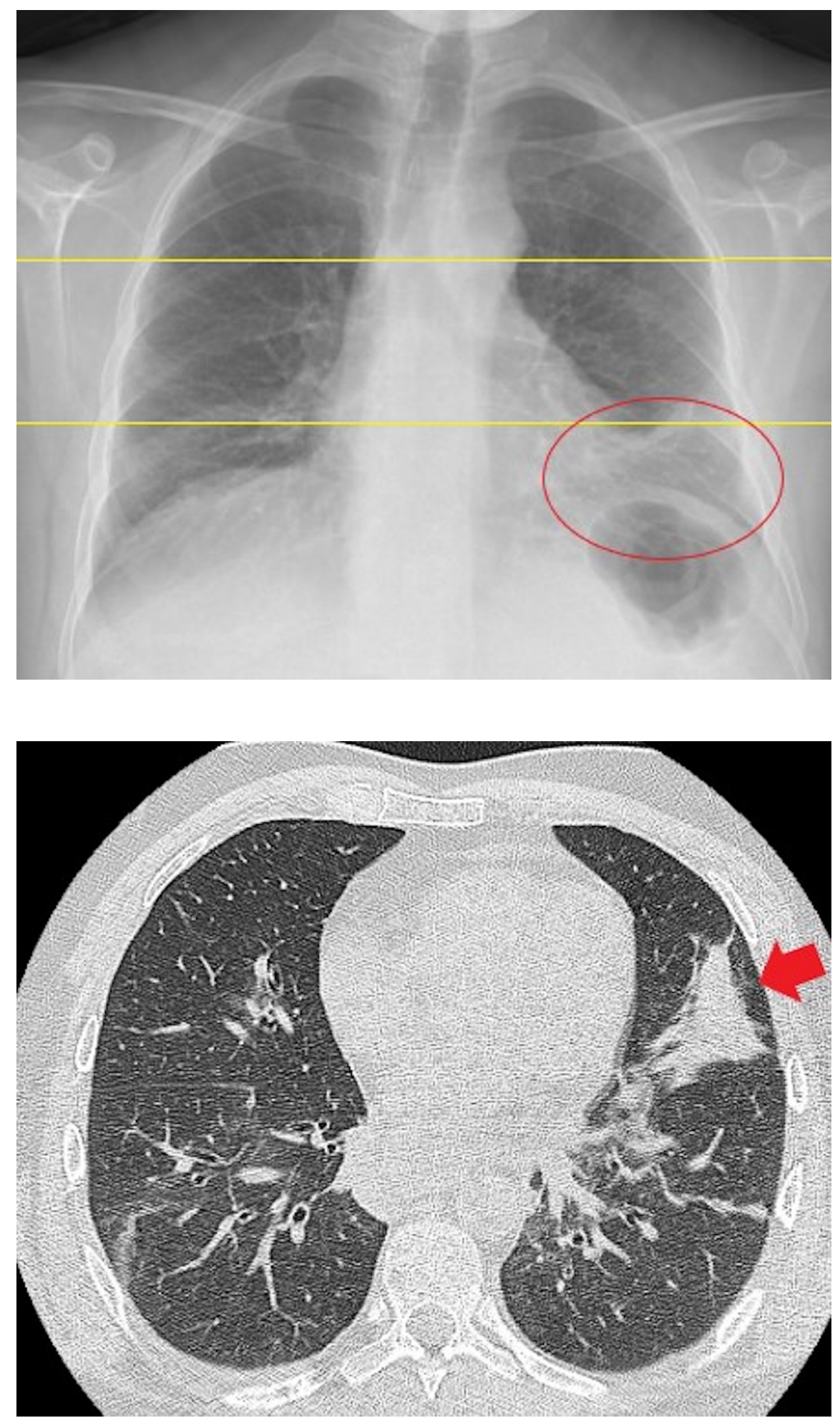